\title{
Erratum
}

\section{The Bando-Calabi-Futaki character as an obstruction to semistability}

\section{T. Mabuchi $\cdot$ Y. Nakagawa}

Math. Ann., http://dx.doi.org/10.1007/s002080200336

Received: 19 November 2003 / Revised version: 11 May 2004 /

Published online: 14 September 2004 - (C) Springer-Verlag 2004

Abstract. The purpose of this note is to correct some errors in (b) and (c) of Main Theorem in [MN], and to make an additional observation. In particular, we shall give a first nonvanishing example of the obstruction $\operatorname{Obstr}_{\text {asymp }}(M, L)$ introduced in [M2].

\section{Corrections}

In this note, we use the same notation as in [MN]. Fix once for all a compact connected $n$-dimensional complex manifold $M$ with an integral Kähler class $\eta$, a linear algebraic group $G$ defined over $\mathbb{C}$ acting biregularly on $M$, and an ample $G$-linearized holomorphic line bundle $L$ over $M$ such that $c_{1}(L)=\eta$. For an ample $G$-linearized holomorphic line bundle $N$ over $M$ and a positive integer $m \in \mathbb{Z}$ such that $N^{m}$ is very ample, we put

$$
V_{N^{m}}:=H^{0}\left(M, N^{m}\right)^{*}, \quad W_{N^{m}}:=\left\{S^{d}\left(V_{N^{m}}\right)\right\}^{\otimes n+1},
$$

where $d=d\left(N^{m}\right)$ is the degree of $M \subset \mathbb{P}\left(V_{N^{m}}\right)$. For the dual $V_{N^{m}}^{*}$ of the complex vector space $V_{N^{m}}$, we naturally have an algebraic group homomorphism

$$
v_{N^{m}}: G \rightarrow \mathrm{GL}\left(V_{N^{m}}^{*}\right) \text {. }
$$

Define a positive integer $\beta=\beta\left(N^{m}\right)$ by setting $\beta:=\operatorname{dim}_{\mathbb{C}} V_{N^{m}}$. Then $v_{N^{m}}$ induces an algebraic character det $v_{N^{m}}: G \rightarrow \mathrm{GL}\left(\wedge^{\beta} V_{N^{m}}^{*}\right) \cong \mathbb{C}^{*}$, and let $\left(\operatorname{det} v_{N^{m}}\right)_{*}: \mathfrak{g} \rightarrow \mathbb{C}$ be the associated Lie algebra character. For a suitable finite

\section{T. MABUCHI}

Department of Mathematics, Osaka University, Toyonaka, Osaka 560-0043, Japan (e-mail: mabuchi@math.sci.osaka-u.ac.jp)

\section{Y. NAKAGAWA}

Department of Mathematics, Kanazawa University, Kakuma-machi, Kanazawa, 920-1192, Japan (e-mail: yasunaka@kenroku.kanazawa-u.ac.jp) 
unramified cover $\iota: \tilde{G} \rightarrow G$ of $G$, the composite $\left(\operatorname{det} v_{N^{m}}\right) \circ \iota$ is expressible as a multiple $\left(\chi_{N^{m}}\right)^{\beta}$ for some algebraic character $\chi_{N^{m}}: \tilde{G} \rightarrow \mathbb{C}^{*}$ of $\tilde{G}$. In particular,

$$
\left(\operatorname{det} v_{N^{m}}\right)_{*}=\beta \cdot\left(\chi_{N^{m}}\right)_{*},
$$

where $\left(\chi_{N^{m}}\right)_{*}: \mathfrak{g} \rightarrow \mathbb{C}$ denotes the Lie algebra character associated with $\chi_{N^{m}}$. We now define an algebraic group homomorphism $\tilde{v}_{N^{m}}: \tilde{G} \rightarrow \operatorname{SL}\left(V_{N^{m}}^{*}\right)$ by

$$
\tilde{v}_{N^{m}}(\tilde{g}):=\chi_{N^{m}}(\tilde{g})^{-1}\left(v_{N^{m}} \circ \iota\right)(\tilde{g}), \quad \text { for all } \tilde{g} \in \tilde{G} .
$$

Let $\mu_{N^{m}}: G \rightarrow \mathrm{GL}\left(W_{N^{m}}\right)$ and $\tilde{\mu}_{N^{m}}: \tilde{G} \rightarrow \mathrm{SL}\left(W_{N^{m}}\right)$ be the algebraic group homomorphisms induced naturally by $v_{N^{m}}$ and $\tilde{v}_{N^{m}}$, respectively. Then by (2) and the definition of $W_{N^{m}}$, we obtain

$$
\tilde{\mu}_{N^{m}}(\tilde{g}):=\chi_{N^{m}}(\tilde{g})^{(n+1) d}\left(\mu_{N^{m}} \circ \iota\right)(\tilde{g}), \quad \text { for all } \tilde{g} \in \tilde{G} .
$$

In [MN], the factors $\chi_{N^{m}}(\tilde{g})^{-1}$ and $\chi_{N^{m}}(\tilde{g})^{-(n+1) d}$ in the expression of $\tilde{v}_{N^{m}}(\tilde{g})$ and $\tilde{\mu}_{N^{m}}(\tilde{g})$, respectively, were disregarded by mistake. Let us first consider the case $N=K_{M}^{-1}$. Then in view of (1) above, (c) of Main Theorem in [MN] should be replaced by

Theorem A. Assume that $\eta=c_{1}(M)>0$ with $L=K_{M}^{-1}$. Let $G=\operatorname{Aut}^{0}(M)$, where $G$ acts naturally on $M$. If $M$ is Chow-semistable with respect to $K_{M}^{-m}$ for some $0<m \in \mathbb{Z}$, then $F$ belongs to $\mathbb{Q}\left(\operatorname{det} v_{K_{M}^{-m}}\right)_{*}$.

Similarly, in view of Remark 3.6 in [MN], we next consider the case $N=$ $K_{M} \otimes L^{k}$ with $m=1$. Then (b) of Main Theorem in [MN] should be replaced by the following:

Theorem B. Assume, for some positive integer $\lambda$, the following conditions are satisfied for all integers $k$ with $\lambda \leq k \leq \lambda+2 n+1$ :

(a) $K_{M} \otimes L^{k}$ is very ample,

(b) $M$ is Chow-semistable with respect to $K_{M} \otimes L^{k}$.

Then $F$ belongs to $\Sigma_{k=\lambda}^{\lambda+2 n+1} \mathbb{Q}\left(\operatorname{det} v_{K_{M} \otimes L^{k}}\right)_{*}$.

In Theorem A, recall that $F=-2 \pi(n+1)^{-1} \mathcal{C}\left\{c_{1}^{n+1}, K_{M}^{-1}\right\}=\alpha_{m}\left(\operatorname{det} v_{K_{M}^{-m}}\right)_{*}$ for some $\alpha_{m} \in \mathbb{Q}$, where $\alpha_{m} \neq 0$ as we see later. Then by (a) of Main Theorem in $[\mathrm{MN}]$, we obtain

Corollary C. In Theorem A above, we assume in addition that $\left(M, K_{M}^{-1}\right)$ is semistable in the sense of Tian. Then $\left(\operatorname{det} v_{K_{M}^{-m}}\right)_{*}=0$, i.e., $G=\operatorname{Aut}^{0}(M)$ acts on $H^{0}\left(M, K_{M}^{-m}\right)$ as a subgroup of $\operatorname{SL}\left(H^{0}\left(M, K_{M}^{-m}\right)\right)$.

It is very plausible that the existence of Kähler-Einstein metrics for Fano manifolds implies a very strong stability condition for polarized algebraic manifolds $\left(M, K_{M}^{-1}\right)$. Hence, in view of Corollary $\mathrm{C}$ above, we pose the following:

Conjecture D. If a Fano manifold $M$ admits a Kähler-Einstein metric, then Aut $^{0}(M)$ acts naturally on $H^{0}\left(M, K_{M}^{-m}\right)$ as a subgroup of $\operatorname{SL}\left(H^{0}\left(M, K_{M}^{-m}\right)\right)$ for all $m \gg 1$. 


\section{An additional observation}

In this section, we make the following additional observation. Let $m$ be a positive integer such that $L^{m}$ is very ample and let $[\hat{M}] \in \mathbb{P}\left(W_{L^{m}}\right)$ be the Chow point of the irreducible reduced effective algebraic cycle $M$ on $\mathbb{P}\left(V_{L^{m}}\right)$, where $[\hat{M}]$ is the natural image of $0 \neq \hat{M} \in W_{L^{m}}$ in $\mathbb{P}\left(W_{L^{m}}\right)$. Let $G$ be the maximal connected linear algebraic subgroup of $\operatorname{Aut}^{0}(M)$, so that $\operatorname{Aut}^{0}(M) / G$ is an Abelian variety. Then $G$ is known to be an algebraic subgroup of $\operatorname{PGL}\left(V_{L^{m}}^{*}\right)$ by a natural inclusion:

$$
G \hookrightarrow \operatorname{PGL}\left(V_{L^{m}}^{*}\right) .
$$

On the other hand, the $G$-linearization of $L$ allows us to regard $G$ as an algebraic subgroup of $\mathrm{GL}\left(V_{L^{m}}^{*}\right)$, and the corresponding inclusion

$$
j: G \hookrightarrow \operatorname{GL}\left(V_{L^{m}}^{*}\right)
$$

is written as $v_{L^{m}}$ by the notation in Section 1 . Next, let $\tilde{G}$ be the identity component of the isotropy subgroup of $\operatorname{SL}\left(V_{L^{m}}^{*}\right)$ at $[\hat{M}]$ for the natural $\operatorname{SL}\left(V_{L^{m}}^{*}\right)$-action on $\mathbb{P}\left(W_{L^{m}}\right)$. Let $\varphi: \operatorname{SL}\left(V_{L^{m}}^{*}\right) \rightarrow \operatorname{PGL}\left(V_{L^{m}}^{*}\right)$ be the natural isogeny. Then by the definition of $\tilde{G}$, we see that $\tilde{G}$ and the identity component of $\varphi^{-1}(G)$ coincide for (4) above. Hence the natural inclusion

$$
\tilde{j}: \tilde{G} \hookrightarrow \operatorname{SL}\left(V_{L^{m}}^{*}\right)
$$

is expressible as $\tilde{v}_{L^{m}}$ by the notation in Section 1, because the representations $j \circ \varphi$ and $\tilde{j}$ of $G$ differ only by a character of $\tilde{G}$.

Assume now that $M$ is Chow-semistable with respect to $L^{m}$. Let $\left(\tilde{\mu}_{L^{m}} \mid \mathbb{C} \cdot \hat{M}\right)_{*}$ : $\mathfrak{g} \rightarrow \mathbb{C}$ denote the Lie algebra character associated with the isotropy representation

$$
\tilde{G} \times \mathbb{C} \cdot \hat{M} \rightarrow \mathbb{C} \cdot \hat{M}, \quad(\tilde{g}, \ell) \mapsto\left\{\tilde{\mu}_{L^{m}}(\tilde{g})\right\}(\ell)
$$

of $\tilde{G}$ at $[\hat{M}]$. Similarly, we have the Lie algebra character $\left(\mu_{L^{m} \mid \mathbb{C} \cdot \hat{M}}\right)_{*}: \mathfrak{g} \rightarrow \mathbb{C}$. By virtue of the $G$-linearization of $L$, we see from (3.5) in [MN] that the equalities $\left(\mu_{L^{m}} \mid \mathbb{C} \cdot \hat{M}\right)_{*}=2 \mathcal{C}\left\{c_{1}^{n+1} ; L^{m}\right\}=2 m^{n+1} \mathcal{C}\left\{c_{1}^{n+1} ; L\right\}$ hold, while the Chow-semistability implies that the isotropy representation is trivial, i.e., $\left(\tilde{\mu}_{L^{m}} \mid \mathbb{C} \cdot \hat{M}\right)_{*}=0$. Now by (2) and (3), we have $\left(\tilde{\mu}_{L^{m}}\right)_{*}=\left(\mu_{L^{m}}\right)_{*}+\beta\left(L^{m}\right)^{-1}(n+1) d\left(L^{m}\right)\left(\operatorname{det} v_{L^{m}}\right)_{*}$. Hence, in view of the identity $d\left(L^{m}\right)=m^{n} c_{1}(L)^{n}[M]$, we see that

$$
\frac{\left(\operatorname{det} v_{L^{m}}\right)_{*}}{m \beta\left(L^{m}\right)}=\frac{\left\{\left(\tilde{\mu}_{L^{m}} \mid \mathbb{C} \cdot \hat{M}\right)_{*}-\left(\mu_{L^{m}} \mid \mathbb{C} \cdot \hat{M}\right)_{*}\right\}}{m(n+1) d\left(L^{m}\right)}=\frac{-2 \mathcal{C}\left\{c_{1}^{n+1}, L\right\}}{(n+1) c_{1}(L)^{n}[M]} .
$$

Here the right-hand side is a Lie algebra character of $\mathfrak{g}$ independent of the choice of $m$, and will be denoted by $\mathcal{F}_{0}$. Thus, we obtain

Theorem E. If $M$ is Chow-semistable with respect to $L^{m}$, then $\left\{m \beta\left(L^{m}\right)\right\}^{-1}$ $\left(\operatorname{det} v_{L^{m}}\right)_{*}$ coincides with $\mathcal{F}_{0}$.

By this theorem applied to the case $L=K_{M}^{-1}$, we immediately obtain Theorem A and the nonvanishing of $\alpha_{m}$ above. 


\section{An example of $(M, L)$ such that $O b s t r_{\text {asymp }}(M, L) \neq 0$}

In this section, we assume for simplicity that $L$ is very ample. Consider the infinitesimal action $\left(v_{L}\right)_{*}: \mathfrak{g} \rightarrow \operatorname{End}\left(V_{L}^{*}\right)$ of $\mathfrak{g}$ on $V_{L}^{*}$ induced by the $G$-linearization of $L$. Put

$$
\rho_{m}:=\left(v_{L}\right)_{*}-m^{-1}\left(\chi_{L^{m}}\right)_{*} \operatorname{id}_{V_{L}^{*}}=\left(v_{L}\right)_{*}-\left\{m \beta\left(L^{m}\right)\right\}^{-1}\left(\operatorname{det} v_{L^{m}}\right)_{*} \operatorname{id}_{V_{L}^{*}} .
$$

As seen from Theorem $\mathrm{E}$ above, vanishing of the obstruction $\operatorname{Obstr}_{\text {asymp }}(M, L)$ to asymptotic Chow-semistability for $(M, L)$ introduced in [M2] is expressible as the stability

$$
\rho_{m_{0}}=\rho_{m_{0}+1}=\rho_{m_{0}+2}=\ldots
$$

of the actions $\rho_{m}, m \gg 1$, or equivalently, as the coincidence of $\left\{m \beta\left(L^{m}\right)\right\}^{-1}$ $\left(\operatorname{det} v_{L^{m}}\right)_{*}$ with $\mathcal{F}_{0}$ for all sufficiently large integers $m$.

We shall now give an example of $(M, L)$ such that $\operatorname{Obstr}_{\text {asymp }}(M, L) \neq 0$. Let $M$ be $\mathbb{P}^{2}(\mathbb{C})$ blown up at a point, and let $L=K_{M}^{-1}>0$. Then there exists an extremal Kähler metric $\omega$ on $M$ in the anticanonical class, and $M$ as a toric variety admits an almost homogeneous action of $T:=\mathbb{C}^{*} \times \mathbb{C}^{*}$. Let $\mathfrak{t}_{\mathbb{R}}$ be the Lie algebra of the maximal compact subgroup $T_{\mathbb{R}}:=S^{1} \times S^{1}$ of $T$. Let $m$ be an arbitrary positive integer. Then for a suitable choice of a basis for $t_{\mathbb{R}}^{*}$, the image of the moment map $\mu: M \rightarrow \mathfrak{t}_{\mathbb{R}}^{*}$ for the Kähler metric $m \omega$ is a compact convex polygon $P$ with integral vertices $(-m, m),(0, m),(2 m,-m),(-m,-m)$ (see for instance [M1]). Let $b_{m}$ be the barycenter of the polygon $P$ and let $b_{m}^{\prime}$ be the barycenter of the set of all integral points in $P$. Then by setting $q(m):=2(m+1)(2 m+1)^{-1}$, we see that

$$
b_{m}=(m / 12,-m / 6) \quad \text { and } \quad b_{m}^{\prime}=q(m)(m / 12,-m / 6) .
$$

Note that $\left\{m \beta\left(L^{m}\right)\right\}^{-1}\left(\operatorname{det} v_{L^{m}}\right)_{*}=\mathcal{F}_{0}$ if and only if $b_{m}^{\prime}=b_{m}$. By $q(m)>1$, we obtain $b_{m} \neq b_{m}^{\prime}$ for all positive integers $m$. Thus, we obtain $O b s t r_{\text {asymp }} \neq 0$ in this case.

Added in proof. After the completion of this note, we hear that the vanishing of the obstruction $\operatorname{Obstr}_{\text {asymp }}(M, L)$ is characterized by Futaki [F1] as the vanishing of a series of integral invariants (including the Bando-Calabi-Futaki character $F$ ) by virtue of the equivariant Riemann-Roch theorem.

\section{References}

[F1] Futaki, A.: Asymptotic Chow stability and integral invariants. Preprint

[M1] Mabuchi, T.: Einstein-Kähler forms, Futaki invariants and convex geometry on toric Fano varieties. Osaka J. Math. 24, 705-737 (1987)

[M2] Mabuchi, T.: An obstruction to asymptotic semistability and approximate critical metrics. arXiv:math. DG/0404210, to appear in Osaka J. Math. 41, 463-472 (2004)

[MN] Mabuchi, T., Nakagawa, Y.: The Bando-calabi-Futaki character as an obstruction to semistability. Math. Ann. 324, 187-193 (2002) 